\title{
Pulsar spectra of radio emission
}

\author{
O. Maron ${ }^{1}$, J. Kijak ${ }^{1}$, M. Kramer ${ }^{2,3}$, and R. Wielebinski ${ }^{2}$ \\ 1 J. Kepler Astronomical Center, Pedagogical University, Lubuska 2, PL-65-265 Zielona Góra, Poland \\ 2 Max-Planck-Institut für Radioastronomie, Auf dem Hügel 69, D-53121 Bonn, Germany \\ ${ }^{3}$ University of Manchester, Jodrell Bank Observatory, Macclesfield, Cheshire SK11 9DL, UK
}

Received April 11; accepted September 7, 2000

\begin{abstract}
We have collected pulsar flux density observations and compiled spectra of 281 objects. The database of Lorimer et al. (1995) has been extended to frequencies higher than $1.4 \mathrm{GHz}$ and lower than $300 \mathrm{MHz}$. Our results show that above $100 \mathrm{MHz}$ the spectra of the majority of pulsars can be described by a simple power law with average value of spectral index $\langle\alpha\rangle=-1.8 \pm 0.2$. A rigorous analysis of spectral fitting revealed only about $10 \%$ of spectra which can be modelled by the two power law. Thus, it seems that single power law is a rule and the two power law spectrum is a rather rare exception, of an unknown origin, to this rule. We have recognized a small number of pulsars with almost flat spectrum $(\alpha \geq-1.0)$ in the wide frequency range (from $300 \mathrm{MHz}$ to $20 \mathrm{GHz}$ ) as well as few pulsars with a turn-over at unusually high frequency $(\sim 1 \mathrm{GHz})$.
\end{abstract}

Key words: pulsars: general — radiation mechanisms: non-thermal

\section{Introduction}

One of the main observables of pulsar emission is its flux density $S_{\nu}$, measured at a given central frequency $\nu$ of the receiver bandwidth. Flux measurements are crucial for deriving, the so called, pulsar luminosity function and therefore the birth rate of the Galactic population of neutron stars. The first spectrum of a pulsar taken at 5 frequencies was published by Robinson et al. (1968). The flux density variations and spectra for frequencies between 0.15 and $1.6 \mathrm{GHz}$ were later reported for a number of pulsars by McLean (1973). Spectra of 27 pulsars were published by Sieber (1973) who used pulse energy values obtained with the $100-\mathrm{m}$ and $25-\mathrm{m}$ telescopes of the Max-Planck-Institute for Radioastronomy (MPIfR) as

Send offprint requests to: O. Maron,

e-mail: olaf@astro.ca.wsp.zgora.pl well as other values published in the literature. He was the first to show a turn-over behaviour at low frequencies around $100 \mathrm{MHz}$ and a break in spectrum at high frequencies (about $1 \mathrm{GHz}$ ). Sieber et al. (1975) published pulse shapes and their energies for 35 pulsars at 2.7 and $4.9 \mathrm{GHz}$, and for 7 pulsars at $10.7 \mathrm{GHz}$. Izvekova et al. (1981) and Slee et al. (1986) presented an analysis of flux measurements for pulsars at meter wavelengths $(\sim 80 \mathrm{MHz})$. A compilation of spectra of 45 pulsars over a wide frequency range was published for the first time by Malofeev et al. (1994). Seiradakis et al. (1995) published a collection of high frequency data on pulsar profiles and flux densities for 183 pulsars at $1.4 \mathrm{GHz}, 46$ pulsars at $4.85 \mathrm{GHz}$ and 24 pulsars at 10.5 GHz. The catalogue of pulsar flux density measurements for 281 pulsars at frequencies ranging from $0.4 \mathrm{GHz}$ to $1.6 \mathrm{GHz}$ was published by Lorimer et al. (1995). The first spectra for four millisecond pulsars were published by Foster et al. (1991). Kramer et al. (1998) presented the results of flux density measurements for 23 millisecond pulsars at frequencies 1.4 and $1.7 \mathrm{GHz}$ and an analysis of their spectra. Van Ommen et al. (1997) presented polarimetric data together with flux density measurements for a large number of southern pulsars at frequencies of $800 \mathrm{MHz}$ and $950 \mathrm{MHz}$. Most recently, Toscano et al. (1998) have presented flux density measurements for southern millisecond and slow pulsars at frequencies between $0.4 \mathrm{GHz}$ and $1.6 \mathrm{GHz}$, and Kramer et al. (1999) studied the emission properties of millisecond pulsars up $4.85 \mathrm{GHz}$. A first large sample of flux densities of weak pulsars at $4.85 \mathrm{GHz}$ was published by Kijak et al. (1998).

It has been long believed that flux density spectra for most of the pulsars have been well described by a simple power law $S \propto \nu^{\alpha}$ with spectral index of $\sim-1.6$ (Sieber 1973; Lorimer et al. 1995). However, a considerable fraction of pulsars demonstrated spectra that required modelling by two power laws (Sieber 1973; Malofeev et al. 1994). These were commonly called the broken-type spectra. In this paper we show that the two power law spectra 
are rare exception and that majority of pulsars can be modelled by a single power law. Our analysis shows that only $10 \%$ of pulsars requires two power law spectra.

Recently, pulsar flux density measurements have been extended to the mm-wavelengths region, which provided information about this newly explored spectral region. These measurements suggested that spectrum flattens out or even turns up at very high frequencies (Kramer et al. 1996). In this paper we present a new and most complete compilation of spectra for 281 pulsars in the frequency range from $39 \mathrm{MHz}$ to $43 \mathrm{GHz}$. The flux density measurements for frequencies from $300 \mathrm{MHz}$ to $1.4 \mathrm{GHz}$ were done at Jodrell Bank (Lorimer et al. 1995). For frequencies equal or above $1.4 \mathrm{GHz}$ we have utilized flux measurements made by different authors at the Effelsberg Radiotelescope of the Max-Planck Institute for Radioastronomy (Sieber et al. 1975; Bartel et al. 1978; Sieber \& Wielebinski 1987; Wielebinski et al. 1993; Seiradakis et al. 1995; Kramer et al. 1996; von Hoensbroech et al. 1997; Kramer et al. 1997; Kijak et al. 1998). We have also reduced a significant amount of the unpublished data available at the archives of the MPIfR. Most of these data is available throughout the European Pulsar Network Database (Lorimer et al. 1998). We have also included the data published by Izvekova et al. (1981) and Malofeev et al. (2000) containing observations made at low radio frequencies from $39 \mathrm{MHz}$ to $102.5 \mathrm{MHz}$. Our own observations at $4.85 \mathrm{GHz}$ made in Effelsberg in 1998 are also included.

\section{Observations and data reduction}

As a basis for our database we have taken the flux density measurements published by Lorimer et al. (1995). These observations were made between July 1988 and October 1992 using the 76-m Lovell radio telescope at Jodrell Bank, at frequencies 408, 606, 925, 1408 and $1606 \mathrm{MHz}$. Lorimer et al. (1995) excluded from their sample those pulsars which were too weak to obtain reliable flux density measurements as well as the millisecond pulsars. We have extended this database by observations made at higher frequencies by different authors mentioned earlier or those unpublished but made available at MPIfR archives in Bonn. All observations at frequency range from $1.4 \mathrm{GHz}$ to $43 \mathrm{GHz}$ were made with the $100-\mathrm{m}$ radio telescope of the MPIfR at Effelsberg and are available in European Pulsar Network Database (Lorimer et al. 1998). Some values at 1.4 and $1.6 \mathrm{GHz}$ were also published by Lorimer et al. (1995). We performed additional observations at $4.85 \mathrm{GHz}$ of 43 very weak pulsars in August 1998. We managed to detect 30 objects and those undetected are listed in Table 1. The detection limit of $S \sim 0.05 \mathrm{mJy}$ for the survey published by Kijak et al. (1998) is clearly visible from this table. The observations published by Izvekova et al. (1981) and Malofeev et al. (2000) were performed
Table 1. Pulsars not detected at $4.85 \mathrm{GHz}$. The pulsar name, total observing time and upper limits $S_{\max }$ for the total flux density are listed

\begin{tabular}{llllll}
\hline PSR B & $\begin{array}{l}\text { Time } \\
{[\mathrm{min}]}\end{array}$ & $\begin{array}{l}S_{\max } \\
{[\mathrm{mJy}]}\end{array}$ & PSR B & $\begin{array}{l}\text { Time } \\
{[\mathrm{min}]}\end{array}$ & $\begin{array}{l}S_{\max } \\
{[\mathrm{mJy}]}\end{array}$ \\
\hline $0621-04$ & 30 & 0.02 & $1820-14$ & 20 & 0.02 \\
$1246+22$ & 50 & 0.01 & $1834-04$ & 30 & 0.01 \\
$1534+12$ & 20 & 0.01 & $1839-04$ & 25 & 0.03 \\
$1600-27$ & 25 & 0.01 & $1848+12$ & 20 & 0.01 \\
$1811+40$ & 20 & 0.01 & $2210+29$ & 100 & 0.01 \\
$1813-17$ & 25 & 0.01 & $2323+63$ & 20 & 0.03 \\
\hline
\end{tabular}

at the Pushchino Radio Astronomical Observatory of the Lebedev Physical Institute.

\subsection{Calibration procedure}

In order to calibrate the flux density of a pulsar using a system in the Effelsberg Radio-observatory, a noise diode installed in every receiver is switched synchronously with the pulse period. The energy output for the noise diode is then compared with energy received from the pulsar, since the first samples of an observed pulse profile contain the calibration signal while the remaining samples contain the pulse. The energy of the noise diode itself can be calibrated by comparing its output to the flux density of a known continuum sources. This pointing procedure is generally performed on well known reliable flux calibrators, e.g. 3C 123, 3C 48, etc. From these pointing observations a factor $f_{\mathrm{c}}$ translating the height of the calibration signal into flux units, is derived. The energy of a pulse is given as the integral beneath its waveform which in arbitrary units yields

$E=\sum_{i \in \text { pulse }} A_{i} \Delta t_{\mathrm{samp}}=\Delta t_{\mathrm{samp}} \sum_{i \in \text { pulse }} A_{i}$

where $A_{i}$ is the pulse amplitude measured in the phase bin $i$ and $\Delta t_{\text {samp }}$ is the sampling time. Scaling the whole profile now in units of the height of the calibration signal measured in the same profile, $A_{\text {cal }}$, we obtain

$E=\Delta t_{\text {samp }} \sum_{i \in \text { pulse }} \frac{A_{i}}{A_{\text {cal }}}$.

Using the conversion factor $f_{\mathrm{c}}$, the mean pulse energy is translated into proper units, i.e. $\mathrm{J} \mathrm{m}^{-2} \mathrm{~Hz}$, according to the following formula

$E=f_{\mathrm{c}} \cdot \frac{\Delta t_{\text {samp }}}{A_{\text {cal }}} \sum_{i \in \text { pulse }} A_{i}$.

The mean flux represents the pulse energy averaged over a pulse period,

$S_{\text {mean }}=\frac{E}{P}$.

Finally, assuming that $f_{\mathrm{c}}$ converts the units of the calibration signal strength into mJy, and the sampling time 
$\Delta t_{\text {samp }}$ is given in $\mu \mathrm{s}$, the mean flux is obtained as (Kramer 1995)

$S_{\text {mean }}=10^{-3} \cdot \frac{f_{\mathrm{c}}}{P} \cdot \frac{\Delta t_{\mathrm{samp}}}{A_{\mathrm{cal}}} \sum_{i \in \text { pulse }} A_{i}$.

\subsection{Error analysis}

Pulsars are generally known to be stable radio sources although the measured flux density varies due to diffractive and refractive scintillation effects (e.g. Stinebring \& Condon 1990). Interstellar scintillations are caused by irregularities in the electron density of the interstellar medium. The observed flux variations are frequency and distance dependent and also depend on the observing set-up, i.e. on the relative width of observing and scintillation bandwidth (e.g. Malofeev et al. 1996; Malofeev 1996). Unless the receiver bandwidth is significantly larger than the scintillation bandwidth, which increases with frequency, strong variations in the observed flux densities are to be expected. Usually, however, the amplitude of scintillation decreases towards higher frequencies, so that those data are less influenced by the scintillation effects. Nevertheless, the question of intrinsic variations on very short and very long time scales remains still open (cf. Stinebring \& Condon 1990). Assessing the situation is hampered by the fact that many authors do not estimate the always present influence of interstellar scintillations or do not quote error estimates at all. Given the difference in the observing set-ups for given observatories, a careful analysis is difficult. We try to circumvent this unpleasant situation by estimating errors for the pulsar flux densities in our sample from published values, wherever available, and standard deviations of the average of single measurements. If only one measurement was available, an error estimate could not be computed although it may happen that form of the spectrum changes when new measurements are added.

\subsection{Search technique for break frequency}

Since a robust theory of pulsar radio emission does not exist, the true shape of pulsar spectra is still not known. A fair first attempt is to model them by simple power laws. Previous studies (e.g. Sieber 1973; Malofeev et al. 1994) showed however that some pulsar spectra cannot apparently be described by this simple approach. Usually, such a conclusion is reached after a visual inspection of the data, i.e. after a power law fit has been done. However, if pulsar spectra are indeed more complicated, the usual next step to fit a composite (or "broken") power law is just another approximation, where the undersampling of the spectrum in the observed range of frequencies would place any fitted "break" naturally in the range of a few $\mathrm{GHz}$, i.e. the range where they are indeed usually observed as it was clearly pointed out by Thorsett (1991). Nevertheless, even if two power laws are just another approximation to a "true" spectrum, any need to fit a break in order to describe the data adequately would represent a valuable hint on the true nature of pulsar spectra. It is therefore very important to search for such breaks in the spectra, while keeping just discussed limitations in mind. We believe, however, that one has to be more quantitative when describing the need for fitting a two power laws rather than a simple power law. This is even more important in the light of the latest results on millisecond pulsar spectra (Kramer et al. 1999; Kuzmin \& Losovski 2000), where no significant break (not even a low frequency turn-over) has been found. Hence, we adopted in this work the following approach: firstly, we fitted a simple power law to the flux density data, assuming that this describes the data sufficiently. We calculated a $\chi^{2}$ and the probability $Q$ that a random $\chi^{2}$ exceeds this value for a given number of degrees of freedom. These computed probabilities give a quantitative measure for the goodness-of-fit of the model. If $Q$ is very small then the apparent discrepancies are unlikely to be chance fluctuations. Much more probably either the model is wrong, or the measurement errors are larger than stated, or measurement errors might not be normally distributed. Generally, one may accept models with $Q \sim 0.001$ (Press et al. 1996). Secondly, we assumed that a two power law had to be fitted to the data, using the following rules:

$S(\nu)= \begin{cases}c_{1} \nu^{\alpha_{1}}: & \nu \leq \nu_{\mathrm{b}} \\ c_{2} \nu^{\alpha_{2}}: & \nu>\nu_{\mathrm{b}}\end{cases}$

Since the break frequency, $\nu_{\mathrm{b}}$, is a priori unknown, we treated it as a free parameter and tried to minimize a corresponding $\chi^{2}$ simultaneously over the whole parameter space of $c_{1}, c_{2}, \alpha_{1}, \alpha_{2}$ and $\nu_{\mathrm{b}}$. Due to the nature of the additional boundary condition $\left(\nu \leq \nu_{\mathrm{b}}\right.$ or $\left.\nu>\nu_{\mathrm{b}}\right)$, we applied a Simplex algorithm as described by Nelder \& Mead (1965). For the resulting, minimized $\chi^{2}$ we then calculated again the probability that a random $\chi^{2}$ is larger than the found value. A comparison of the $\chi^{2}$-statistics for both cases was then used to judge whether a break was truly significant or not. The fitting procedure was performed on data in the frequency range from $400 \mathrm{MHz}$ to $23 \mathrm{GHz}$. We have not taken into account data corresponding to single measurements, as well as those at frequencies lower than $400 \mathrm{MHz}$, as they could represent a low frequency turnover which usually occurs at $\sim 100 \mathrm{MHz}$. There is a gap in data coverage between 100 and $300 \mathrm{MHz}$, but this should not affect our analysis and conclusions.

\section{Results and discussion}

In this paper we obtained a large database of flux density measurements over a wide range of frequencies, from $39 \mathrm{MHz}$ to $43 \mathrm{GHz}$. Although measurements were made 
Table 2. Spectral indices for 266 pulsars with simple power-law spectrum $S \sim \nu^{\alpha}$ calculated for a given frequency range with error and a probability of the goodness-of-fit $Q$ (see text)

\begin{tabular}{|c|c|c|c|c|c|c|c|c|c|}
\hline PSR B & $\begin{array}{l}\text { Freq. range } \\
\text { [GHz] }\end{array}$ & $\alpha$ & $\sigma_{\alpha}$ & $\bar{Q}$ & PSR B & Freq. range & $\alpha$ & $\sigma_{\alpha}$ & $Q$ \\
\hline $0011+47$ & $0.4-4.9$ & -1.3 & 0.10 & $8.3 \mathrm{E}-01$ & $0950+08$ & $0.4-10.6$ & -2.2 & 0.03 & $1.7 \mathrm{E}-02$ \\
\hline $0031-07$ & $0.4-10.7$ & -1.4 & 0.11 & $4.1 \mathrm{E}-03$ & $1010-23$ & $0.4-0.6$ & -1.9 & & \\
\hline $0037+56$ & $0.4-4.8$ & -1.8 & 0.05 & 6.3E-03 & $1016-16$ & $0.4-1.4$ & -1.7 & 0.28 & $9.1 \mathrm{E}-01$ \\
\hline $0045+33$ & $0.4-1.4$ & -2.5 & 0.26 & & $1039-19$ & $0.4-1.4$ & -1.5 & 0.28 & $6.8 \mathrm{E}-01$ \\
\hline $0052+51$ & $0.4-1.4$ & -0.7 & 0.14 & $6.1 \mathrm{E}-01$ & $1112+50$ & $0.4-4.9$ & -1.7 & 0.11 & $6.0 \mathrm{E}-02$ \\
\hline $0053+47$ & $0.4-4.9$ & -1.6 & 0.09 & & $1133+16$ & $0.4-32.0$ & -1.9 & 0.06 & $7.4 \mathrm{E}-02$ \\
\hline $0059+65$ & $0.4-1.6$ & -1.6 & 0.13 & $1.4 \mathrm{E}-01$ & $1254-10$ & $0.4-1.6$ & -1.8 & 0.16 & $6.4 \mathrm{E}-01$ \\
\hline $0105+65$ & $1.4-1.4$ & -1.9 & 0.19 & $4.3 \mathrm{E}-01$ & $1309-12$ & $0.4-1.4$ & -1.7 & 0.16 & $2.8 \mathrm{E}-01$ \\
\hline $0105+68$ & $0.4-1.4$ & -1.8 & 0.22 & & $1322+83$ & $0.4-1.4$ & -1.6 & 0.30 & 8.7E-01 \\
\hline $0114+58$ & $0.4-1.4$ & -2.5 & 0.21 & $8.5 \mathrm{E}-01$ & $1508+55$ & $0.4-4.9$ & -2.2 & 0.07 & $5.2 \mathrm{E}-02$ \\
\hline $0138+59$ & $0.4-1.4$ & -1.9 & 0.16 & 7.0E-01 & $1530+27$ & $0.4-4.9$ & -1.4 & 0.10 & $1.3 \mathrm{E}-01$ \\
\hline $0144+59$ & $0.4-14.6$ & -1.0 & 0.04 & $6.1 \mathrm{E}-04$ & $1540-06$ & $0.4-4.9$ & -2.0 & 0.11 & $4.0 \mathrm{E}-02$ \\
\hline $0148-06$ & $0.4-1.4$ & -2.7 & 0.58 & 4.6E-01 & $1541+09$ & $0.4-4.9$ & -2.6 & 0.04 & $2.7 \mathrm{E}-03$ \\
\hline $0149-16$ & $0.4-1.4$ & -2.1 & 0.26 & 2.3E-01 & $1552-23$ & $0.4-4.9$ & -1.8 & 0.08 & $1.1 \mathrm{E}-02$ \\
\hline $0153+39$ & $0.4-0.6$ & -2.2 & & & $1552-31$ & $0.4-1.4$ & -1.6 & 0.19 & 3.0E-04 \\
\hline $0154+61$ & $0.4-1.4$ & -0.9 & 0.12 & $1.4 \mathrm{E}-03$ & $1600-27$ & $0.4-1.4$ & -1.7 & 0.13 & $4.0 \mathrm{E}-04$ \\
\hline $0320+39$ & $0.4-1.4$ & -2.9 & 0.24 & $5.2 \mathrm{E}-01$ & $1604-00$ & $0.4-4.9$ & -1.5 & 0.08 & $6.8 \mathrm{E}-01$ \\
\hline $0329+54$ & $1.4-23.0$ & -2.2 & 0.03 & $7.5 \mathrm{E}-04$ & $1607-13$ & $0.4-0.6$ & -2.1 & 0.45 & \\
\hline $0331+45$ & $0.4-1.4$ & -1.9 & 0.24 & $1.5 \mathrm{E}-01$ & $1612+07$ & $0.4-1.4$ & -2.6 & 0.30 & $1.7 \mathrm{E}-01$ \\
\hline $0339+53$ & $0.4-1.4$ & -2.2 & 0.28 & $2.5 \mathrm{E}-02$ & $1612-29$ & $0.4-0.6$ & -0.8 & 0.98 & \\
\hline $0353+52$ & $0.4-1.4$ & -1.6 & 0.12 & $7.4 \mathrm{E}-01$ & $1620-09$ & $0.4-4.9$ & -1.7 & 0.13 & $1.5 \mathrm{E}-01$ \\
\hline $0402+61$ & $0.4-1.4$ & -1.4 & 0.08 & $1.4 \mathrm{E}-01$ & $1633+24$ & $0.4-1.4$ & -2.4 & 0.31 & \\
\hline $0410+69$ & $0.4-1.4$ & -2.4 & 0.13 & $8.1 \mathrm{E}-04$ & $1642-03$ & $0.4-10.6$ & -2.3 & 0.05 & 6.7E-01 \\
\hline $0447-12$ & $0.4-1.4$ & -2.0 & 0.11 & $1.4 \mathrm{E}-02$ & $1648-17$ & $0.4-1.4$ & -2.5 & 0.26 & $5.9 \mathrm{E}-02$ \\
\hline $0450+55$ & $0.4-4.9$ & -1.5 & 0.04 & $6.0 \mathrm{E}-02$ & $1649-23$ & $0.4-1.4$ & -1.7 & 0.09 & $5.6 \mathrm{E}-01$ \\
\hline $0450-18$ & $0.4-4.9$ & -2.0 & 0.05 & $2.9 \mathrm{E}-08$ & $1657-13$ & $0.4-0.6$ & -1.7 & 0.36 & \\
\hline $0458+46$ & $0.4-1.4$ & -1.3 & 0.05 & $1.2 \mathrm{E}-05$ & $1700-18$ & $0.4-1.4$ & -1.9 & 0.23 & $4.8 \mathrm{E}-05$ \\
\hline $0523+11$ & $0.4-1.4$ & -2.0 & 0.06 & $1.4 \mathrm{E}-01$ & $1700-32$ & $0.4-0.6$ & -3.1 & 0.27 & \\
\hline $0525+21$ & $0.4-4.9$ & -1.5 & 0.12 & $4.4 \mathrm{E}-01$ & $1702-19$ & $0.4-4.9$ & -1.3 & 0.05 & $5.3 \mathrm{E}-01$ \\
\hline $0531+21$ & $0.4-1.4$ & -3.1 & 0.18 & $5.7 \mathrm{E}-01$ & $1706-16$ & $0.4-32.0$ & -1.5 & 0.04 & $1.1 \mathrm{E}-01$ \\
\hline $\mathrm{J} 0538+2817$ & $1.4-4.9$ & -1.2 & 0.57 & & $1709-15$ & $0.4-4.9$ & -1.7 & 0.06 & 8.7E-01 \\
\hline $0559-05$ & $0.4-4.9$ & -1.7 & 0.04 & 7.6E-01 & $1714-34$ & $0.4-1.4$ & -2.6 & 0.34 & \\
\hline $0609+37$ & $0.4-1.4$ & -1.5 & 0.25 & 3.5E-01 & $1717-16$ & $0.4-4.9$ & -2.2 & 0.05 & $5.7 \mathrm{E}-01$ \\
\hline $0611+22$ & $0.4-2.7$ & -2.1 & 0.04 & $8.5 \mathrm{E}-01$ & $1717-29$ & $0.4-1.4$ & -2.2 & 0.20 & $8.8 \mathrm{E}-01$ \\
\hline $0621-04$ & $0.4-1.4$ & -0.4 & 0.29 & $5.0 \mathrm{E}-01$ & $1718-02$ & $0.4-1.4$ & -2.2 & 0.16 & $1.6 \mathrm{E}-06$ \\
\hline $0626+24$ & $0.4-4.9$ & -1.6 & 0.08 & $1.2 \mathrm{E}-03$ & $1718-32$ & $0.4-1.4$ & -2.3 & 0.06 & $3.9 \mathrm{E}-01$ \\
\hline $0628-28$ & $0.4-10.6$ & -1.9 & 0.10 & $6.8 \mathrm{E}-01$ & $1726-00$ & $0.4-0.6$ & -2.3 & 0.47 & \\
\hline $0643+80$ & $0.4-4.9$ & -1.9 & 0.08 & 2.3E-01 & $1727-33$ & $0.4-1.4$ & -1.3 & & \\
\hline $0655+64$ & $0.4-1.4$ & -2.1 & 0.30 & $1.0 \mathrm{E}-01$ & $1730-22$ & $0.4-1.4$ & -2.0 & 0.15 & $1.4 \mathrm{E}-01$ \\
\hline $0656+14$ & $0.4-1.4$ & -0.5 & 0.17 & $1.3 \mathrm{E}-01$ & $1732-07$ & $0.4-1.4$ & -1.9 & 0.12 & $1.4 \mathrm{E}-09$ \\
\hline $0727-18$ & $0.4-1.4$ & -1.6 & 0.11 & $1.3 \mathrm{E}-02$ & $1734-35$ & $0.6-1.4$ & -1.6 & 0.30 & \\
\hline $0740-28$ & $0.4-10.6$ & -2.0 & 0.03 & $1.1 \mathrm{E}-07$ & $1735-32$ & $0.4-1.6$ & -0.9 & 0.12 & $2.1 \mathrm{E}-02$ \\
\hline $0751+32$ & $0.4-4.9$ & -1.5 & 0.07 & $1.8 \mathrm{E}-01$ & $1736-31$ & $0.6-1.6$ & -0.9 & 0.20 & $4.2 \mathrm{E}-01$ \\
\hline $0756-15$ & $0.4-4.9$ & -1.6 & 0.13 & $6.0 \mathrm{E}-04$ & $1737+13$ & $0.4-4.9$ & -1.5 & 0.10 & $2.8 \mathrm{E}-01$ \\
\hline $0809+74$ & $0.4-10.6$ & -1.4 & 0.06 & $6.7 \mathrm{E}-02$ & $1737-30$ & $0.4-1.4$ & -1.3 & 0.10 & $4.5 \mathrm{E}-01$ \\
\hline $0818-13$ & $0.4-4.9$ & -2.3 & 0.05 & $3.4 \mathrm{E}-01$ & $1738-08$ & $0.4-4.9$ & -2.2 & 0.08 & $6.9 \mathrm{E}-02$ \\
\hline $0820+02$ & $0.4-4.9$ & -2.4 & 0.08 & $9.5 \mathrm{E}-01$ & $1740-03$ & $0.4-1.4$ & -1.5 & & \\
\hline $0834+06$ & $0.4-4.9$ & -2.7 & 0.11 & $2.1 \mathrm{E}-02$ & $1740-13$ & $0.4-1.4$ & -2.0 & 0.19 & 2.3E-02 \\
\hline $0853-33$ & $0.4-1.4$ & -2.4 & 0.20 & $5.4 \mathrm{E}-01$ & $1740-31$ & $0.6-1.4$ & -1.9 & 0.11 & \\
\hline $0906-17$ & $0.4-1.4$ & -1.4 & 0.16 & 2.0E-01 & $1742-30$ & $0.4-4.9$ & -1.6 & 0.04 & $1.5 \mathrm{E}-06$ \\
\hline $0917+63$ & $0.4-1.4$ & -1.7 & 0.37 & & $1745-12$ & $0.4-1.4$ & -2.1 & 0.12 & $3.4 \mathrm{E}-03$ \\
\hline $0919+06$ & $0.4-10.6$ & -1.8 & 0.05 & $7.6 \mathrm{E}-01$ & $1746-30$ & $0.4-1.4$ & -1.5 & 0.39 & \\
\hline $0940+16$ & $0.4-1.4$ & -1.3 & 0.30 & $5.3 \mathrm{E}-01$ & $1747-31$ & $0.6-1.4$ & -1.2 & 0.31 & \\
\hline $0942-13$ & $0.4-1.4$ & -3.0 & 0.30 & $8.8 \mathrm{E}-01$ & $1750-24$ & $0.9-4.9$ & -1.0 & 0.07 & $1.1 \mathrm{E}-06$ \\
\hline $0943+10$ & $0.4-0.6$ & -3.7 & 0.36 & & $1753+52$ & $0.4-4.9$ & -1.6 & 0.08 & $5.8 \mathrm{E}-04$ \\
\hline
\end{tabular}


Table 2. continued

\begin{tabular}{|c|c|c|c|c|c|c|c|c|c|}
\hline PSR B & $\begin{array}{l}\text { Freq. range } \\
{[\mathrm{GHz}]}\end{array}$ & $\alpha$ & $\sigma_{\alpha}$ & $Q$ & PSR B & Freq. range & $\alpha$ & $\sigma_{\alpha}$ & $Q$ \\
\hline $1753-24$ & $0.4-1.6$ & -0.7 & 0.14 & $3.2 \mathrm{E}-01$ & $1845-19$ & $0.4-0.6$ & -2.0 & 0.46 & \\
\hline $1754-24$ & $0.4-1.4$ & -1.1 & 0.09 & $9.1 \mathrm{E}-02$ & $1846-06$ & $0.4-1.4$ & -2.2 & 0.10 & $8.4 \mathrm{E}-01$ \\
\hline $1756-22$ & $0.4-4.9$ & -1.7 & 0.09 & $5.3 \mathrm{E}-01$ & $1848+04$ & $0.6-1.4$ & -1.4 & & \\
\hline $1757-24$ & $0.4-0.6$ & -3.6 & & & $1848+12$ & $0.4-1.6$ & -1.9 & 0.16 & $4.3 \mathrm{E}-02$ \\
\hline $1758-03$ & $0.4-1.4$ & -2.6 & 0.11 & $2.3 \mathrm{E}-01$ & $1848+13$ & $0.4-1.6$ & -1.4 & 0.18 & $1.2 \mathrm{E}-01$ \\
\hline $1758-23$ & $1.4-4.9$ & -2.5 & 0.10 & 8.3E-04 & $1849+00$ & $1.4-4.9$ & -2.4 & 0.12 & $4.8 \mathrm{E}-01$ \\
\hline $1800-27$ & $0.4-1.4$ & -1.4 & & & $1851-14$ & $0.4-0.6$ & -0.8 & 0.42 & \\
\hline $1802-07$ & $0.4-1.4$ & -1.3 & 0.31 & & $1853+01$ & $0.4-0.6$ & -2.5 & & \\
\hline $1804-08$ & $0.4-4.9$ & -1.2 & 0.08 & $6.1 \mathrm{E}-07$ & $1855+02$ & $0.6-4.9$ & -1.2 & 0.09 & $9.5 \mathrm{E}-02$ \\
\hline $1804-27$ & $0.6-1.4$ & -3.0 & 0.21 & & $1857-26$ & $0.4-10.7$ & -2.1 & 0.06 & $1.2 \mathrm{E}-03$ \\
\hline $1805-20$ & $0.6-4.9$ & -1.5 & 0.07 & & $1859+01$ & $0.4-0.6$ & -2.9 & 0.21 & \\
\hline $1806-21$ & $0.6-1.6$ & -2.0 & 0.34 & $7.5 \mathrm{E}-02$ & $1859+03$ & $0.4-4.9$ & -2.8 & 0.08 & $3.8 \mathrm{E}-02$ \\
\hline $1810+02$ & $0.4-1.4$ & -1.7 & 0.21 & $3.1 \mathrm{E}-02$ & $1859+07$ & $0.4-1.6$ & -1.0 & 0.15 & $8.2 \mathrm{E}-01$ \\
\hline $1811+40$ & $0.4-1.4$ & -1.8 & 0.22 & $5.0 \mathrm{E}-02$ & $1900+01$ & $0.4-4.9$ & -1.9 & 0.15 & $3.3 \mathrm{E}-01$ \\
\hline $1813-17$ & $0.6-1.6$ & -1.0 & 0.14 & $5.0 \mathrm{E}-01$ & $1900+05$ & $0.4-4.9$ & -1.7 & 0.08 & $3.9 \mathrm{E}-02$ \\
\hline $1813-26$ & $0.4-0.6$ & -1.4 & 0.33 & & $1900+06$ & $0.4-4.9$ & -2.2 & 0.10 & $1.4 \mathrm{E}-01$ \\
\hline $1815-14$ & $0.9-1.6$ & -1.6 & 0.22 & 8.5E-04 & $1900-06$ & $0.4-0.6$ & -1.8 & 0.19 & \\
\hline $1817-13$ & $0.6-1.6$ & -1.7 & 0.37 & $3.7 \mathrm{E}-01$ & $1902-01$ & $0.4-1.4$ & -1.9 & 0.11 & $1.1 \mathrm{E}-02$ \\
\hline $1817-18$ & $0.4-1.4$ & -1.1 & 0.27 & & $1903+07$ & $0.6-1.4$ & -1.3 & 0.10 & $2.6 \mathrm{E}-01$ \\
\hline $1818-04$ & $0.4-4.9$ & -2.4 & 0.06 & $9.7 \mathrm{E}-02$ & $1904+06$ & $0.4-1.6$ & -0.7 & 0.21 & $9.6 \mathrm{E}-01$ \\
\hline $1819-22$ & $0.4-1.4$ & -1.7 & 0.07 & $2.1 \mathrm{E}-01$ & $1905+39$ & $0.4-1.4$ & -2.0 & 0.16 & $9.0 \mathrm{E}-02$ \\
\hline $1820-11$ & $0.4-4.9$ & -1.5 & 0.05 & $1.9 \mathrm{E}-04$ & $1907+00$ & $0.4-1.4$ & -2.0 & 0.11 & $5.6 \mathrm{E}-08$ \\
\hline $1820-14$ & $0.6-1.4$ & -0.7 & 0.22 & & $1907+02$ & $0.4-1.4$ & -2.8 & 0.11 & $3.8 \mathrm{E}-02$ \\
\hline 1820-30B & $0.4-0.6$ & -1.9 & 0.33 & & $1907+03$ & $0.4-4.9$ & -1.8 & 0.07 & $1.4 \mathrm{E}-01$ \\
\hline $1820-31$ & $0.4-1.4$ & -2.1 & 0.20 & $4.8 \mathrm{E}-01$ & $1907+10$ & $0.4-1.4$ & -2.5 & 0.09 & $5.6 \mathrm{E}-01$ \\
\hline $1821+05$ & $0.4-1.4$ & -1.7 & 0.18 & $2.1 \mathrm{E}-02$ & $1907-03$ & $0.4-1.4$ & -2.7 & 0.12 & $7.4 \mathrm{E}-05$ \\
\hline $1821-11$ & $0.6-4.9$ & -2.3 & 0.10 & $1.2 \mathrm{E}-01$ & $1910+20$ & $0.4-1.4$ & -1.6 & 0.16 & 5.9E-01 \\
\hline $1821-19$ & $0.4-4.9$ & -1.9 & 0.06 & $2.2 \mathrm{E}-01$ & $1911+11$ & $0.4-0.6$ & -1.4 & & \\
\hline $1822+00$ & $0.4-1.4$ & -2.4 & 0.26 & $4.0 \mathrm{E}-01$ & $1911+13$ & $0.4-4.9$ & -1.5 & 0.06 & $8.5 \mathrm{E}-03$ \\
\hline $1822-09$ & $0.4-10.6$ & -1.3 & 0.08 & $1.3 \mathrm{E}-02$ & $1911-04$ & $0.4-1.4$ & -2.6 & 0.11 & 3.3E-01 \\
\hline $1822-14$ & $1.4-4.9$ & -0.7 & 0.08 & $3.6 \mathrm{E}-01$ & $1913+10$ & $0.4-1.6$ & -1.9 & 0.15 & $5.6 \mathrm{E}-01$ \\
\hline $1823-11$ & $0.4-1.6$ & -2.4 & 0.10 & $9.9 \mathrm{E}-01$ & $1913+16$ & $0.4-1.4$ & -1.4 & 0.24 & \\
\hline $1823-13$ & $0.6-10.6$ & -0.6 & & & $1913+167$ & $0.4-0.6$ & -1.4 & 0.59 & \\
\hline $1826-17$ & $0.4-4.9$ & -1.7 & 0.06 & $5.1 \mathrm{E}-05$ & $1914+09$ & $0.4-1.4$ & -2.3 & 0.11 & 2.1E-03 \\
\hline $1828-10$ & $0.4-1.6$ & -0.4 & 0.14 & $2.3 \mathrm{E}-01$ & $1914+13$ & $0.4-4.9$ & -1.6 & 0.10 & $3.0 \mathrm{E}-02$ \\
\hline $1829-08$ & $0.4-1.6$ & -0.8 & 0.06 & $1.5 \mathrm{E}-04$ & $1915+13$ & $0.4-4.9$ & -1.8 & 0.09 & 3.7E-02 \\
\hline $1829-10$ & $0.4-1.6$ & -1.3 & 0.15 & $1.4 \mathrm{E}-01$ & $1916+14$ & $0.4-1.4$ & -0.3 & 0.43 & $1.0 \mathrm{E}+00$ \\
\hline $1830-08$ & $0.6-4.9$ & -1.1 & 0.05 & $1.2 \mathrm{E}-21$ & $1917+00$ & $0.4-4.9$ & -2.2 & 0.07 & 6.3E-01 \\
\hline $1831-00$ & $0.4-0.6$ & -1.4 & & & $1918+19$ & $0.4-1.4$ & -2.4 & 0.16 & $9.4 \mathrm{E}-01$ \\
\hline $1831-03$ & $0.4-1.4$ & -2.7 & 0.08 & $1.1 \mathrm{E}-04$ & $1919+14$ & $0.4-4.9$ & -1.3 & 0.14 & $9.2 \mathrm{E}-01$ \\
\hline $1831-04$ & $0.4-4.9$ & -1.3 & 0.07 & $9.5 \mathrm{E}-01$ & $1919+21$ & $0.4-4.9$ & -2.6 & 0.04 & $0.0 \mathrm{E}+00$ \\
\hline $1832-06$ & $0.6-1.6$ & -0.4 & 0.35 & $2.9 \mathrm{E}-01$ & $1920+20$ & $0.4-0.6$ & -2.5 & 0.70 & \\
\hline $1834-04$ & $0.6-1.6$ & -1.9 & 0.30 & $5.8 \mathrm{E}-01$ & $1920+21$ & $0.4-4.9$ & -2.2 & 0.07 & $2.8 \mathrm{E}-02$ \\
\hline $1834-06$ & $0.6-1.6$ & -1.2 & 0.24 & $9.5 \mathrm{E}-01$ & $1923+04$ & $0.4-0.6$ & -2.7 & 0.50 & \\
\hline $1834-10$ & $0.4-1.6$ & -2.1 & 0.09 & $3.1 \mathrm{E}-01$ & $1924+16$ & $0.4-1.4$ & -1.5 & 0.16 & $2.9 \mathrm{E}-01$ \\
\hline $1838-04$ & $0.9-1.6$ & -1.3 & 0.21 & $2.0 \mathrm{E}-01$ & $1929+10$ & $0.4-24$ & -1.6 & 0.04 & $1.5 \mathrm{E}-07$ \\
\hline $1839+09$ & $0.4-1.4$ & -2.0 & 0.07 & $3.1 \mathrm{E}-01$ & $1929+20$ & $0.4-1.4$ & -2.5 & 0.22 & $7.4 \mathrm{E}-01$ \\
\hline $1839+56$ & $0.4-1.4$ & -1.5 & 0.22 & $3.2 \mathrm{E}-01$ & $1930+22$ & $0.4-1.6$ & -1.5 & 0.09 & 3.9E-02 \\
\hline $1839-04$ & $0.4-1.6$ & -1.6 & 0.08 & $1.7 \mathrm{E}-01$ & $1931+24$ & $0.4-0.6$ & -4.0 & & \\
\hline $1841-04$ & $0.4-1.6$ & -1.6 & 0.07 & $5.8 \mathrm{E}-03$ & $1933+16$ & $0.4-4.9$ & -1.7 & 0.03 & 8.1E-03 \\
\hline $1841-05$ & $0.6-4.9$ & -1.7 & 0.10 & $5.3 \mathrm{E}-01$ & $1935+25$ & $0.4-1.4$ & -0.7 & 0.20 & $5.6 \mathrm{E}-02$ \\
\hline $1842+14$ & $0.4-4.9$ & -1.6 & 0.09 & $1.7 \mathrm{E}-02$ & $1937-26$ & $0.4-1.4$ & -0.9 & 0.30 & $1.9 \mathrm{E}-01$ \\
\hline $1842-02$ & $0.6-1.6$ & -0.9 & 0.27 & 4.1E-02 & $1940-12$ & $0.4-1.4$ & -2.4 & 0.20 & $5.1 \mathrm{E}-01$ \\
\hline $1842-04$ & $0.6-1.4$ & -0.8 & 0.29 & $1.4 \mathrm{E}-01$ & $1941-17$ & $0.4-1.4$ & -2.3 & 0.30 & \\
\hline $1844-04$ & $0.4-4.9$ & -2.2 & 0.06 & $8.2 \mathrm{E}-02$ & $1942-00$ & $0.4-1.4$ & -1.8 & 0.17 & $2.5 \mathrm{E}-02$ \\
\hline $1845-01$ & $0.4-10.6$ & -1.6 & 0.05 & 4.0E-01 & $1943-29$ & $0.4-1.4$ & -2.0 & 0.25 & 8.9E-01 \\
\hline
\end{tabular}


Table 2. continued

\begin{tabular}{llllllllll}
\hline PSR B & $\begin{array}{l}\text { Freq. range } \\
\text { [GHz] }\end{array}$ & $\alpha$ & $\sigma_{\alpha}$ & $Q$ & PSR B & Freq. range & $\alpha$ & $\sigma_{\alpha}$ & $Q$ \\
\hline $1944+17$ & $0.4-4.9$ & -1.3 & 0.12 & $1.9 \mathrm{E}-01$ & $2111+46$ & $0.4-10.6$ & -2.1 & 0.04 & $2.1 \mathrm{E}-02$ \\
$1946+35$ & $0.4-4.9$ & -2.4 & 0.04 & $6.9 \mathrm{E}-09$ & $2113+14$ & $0.4-1.4$ & -1.9 & 0.13 & $3.7 \mathrm{E}-02$ \\
$1946-25$ & $0.4-1.4$ & -2.0 & 0.22 & $5.4 \mathrm{E}-01$ & $2148+52$ & $0.4-1.6$ & -1.3 & 0.05 & $1.1 \mathrm{E}-04$ \\
$1951+32$ & $0.4-1.6$ & -1.6 & 0.11 & $5.2 \mathrm{E}-03$ & $2148+63$ & $0.4-2.7$ & -1.8 & 0.09 & $1.1 \mathrm{E}-03$ \\
$1953+50$ & $0.4-4.9$ & -1.6 & 0.09 & $8.5 \mathrm{E}-01$ & $2152-31$ & $0.4-1.4$ & -2.3 & 0.40 & $3.9 \mathrm{E}-01$ \\
$2000+32$ & $0.4-4.9$ & -1.1 & 0.04 & $3.1 \mathrm{E}-02$ & $2154+40$ & $0.4-4.9$ & -1.6 & 0.09 & $3.6 \mathrm{E}-02$ \\
$2000+40$ & $0.4-4.9$ & -2.2 & 0.03 & $5.5 \mathrm{E}-30$ & $2210+29$ & $0.4-1.4$ & -1.5 & 0.20 & $1.3 \mathrm{E}-01$ \\
$2002+31$ & $0.4-1.4$ & -1.7 & 0.06 & $2.3 \mathrm{E}-20$ & $2217+47$ & $0.4-4.9$ & -2.6 & 0.19 & $4.5 \mathrm{E}-01$ \\
$2003-08$ & $0.4-1.4$ & -1.4 & 0.20 & $9.4 \mathrm{E}-02$ & $2224+65$ & $0.4-4.6$ & -1.9 & 0.11 & $3.9 \mathrm{E}-01$ \\
$2016+28$ & $0.4-10.6$ & -2.2 & 0.04 & $4.7 \mathrm{E}-01$ & $2227+61$ & $0.4-1.4$ & -2.6 & 0.10 & $5.7 \mathrm{E}-03$ \\
$2022+50$ & $0.4-4.9$ & -0.8 & 0.05 & $5.3 \mathrm{E}-01$ & $2241+69$ & $0.4-1.4$ & -1.4 & 0.46 & $5.8 \mathrm{E}-01$ \\
$2027+37$ & $0.4-1.4$ & -2.5 & 0.10 & $4.6 \mathrm{E}-02$ & $2255+58$ & $0.4-4.9$ & -2.1 & 0.07 & $1.0 \mathrm{E}+00$ \\
$2035+36$ & $0.4-1.7$ & -1.6 & 0.39 & $9.8 \mathrm{E}-01$ & $2303+30$ & $0.4-1.4$ & -2.3 & 0.16 & $3.6 \mathrm{E}-03$ \\
$2036+53$ & $0.4-1.4$ & -2.0 & 0.27 & $2.2 \mathrm{E}-01$ & $2303+46$ & $0.4-1.6$ & -1.6 & & \\
$2044+15$ & $0.4-1.4$ & -1.7 & 0.15 & $3.4 \mathrm{E}-03$ & $2306+55$ & $0.4-4.9$ & -1.9 & 0.06 & $3.1 \mathrm{E}-01$ \\
$2045+56$ & $0.4-1.4$ & -2.4 & & & $2310+42$ & $0.4-10.6$ & -1.9 & 0.03 & $5.1 \mathrm{E}-05$ \\
$2045-16$ & $0.4-10.6$ & -2.1 & 0.07 & $1.8 \mathrm{E}-01$ & $2315+21$ & $0.4-1.4$ & -2.1 & 0.44 & $6.9 \mathrm{E}-01$ \\
$2053+21$ & $0.4-1.4$ & -0.8 & 0.35 & & $2323+63$ & $0.4-1.4$ & -0.8 & 0.23 & $6.4 \mathrm{E}-02$ \\
$2053+36$ & $0.4-1.4$ & -1.9 & 0.04 & $3.5 \mathrm{E}-03$ & $2327-20$ & $0.4-1.4$ & -2.0 & 0.29 & $8.7 \mathrm{E}-01$ \\
$2106+44$ & $0.4-4.9$ & -1.4 & 0.06 & $7.5 \mathrm{E}-04$ & $2334+61$ & $0.4-1.4$ & -1.7 & 0.23 & $6.3 \mathrm{E}-01$ \\
$2110+27$ & $0.4-1.4$ & -2.2 & 0.18 & $5.6 \mathrm{E}-01$ & $2351+61$ & $0.4-10.6$ & -1.1 & 0.13 & $3.1 \mathrm{E}-01$ \\
\hline
\end{tabular}

Table 3. Spectral indices for 15 pulsars with two-power-law spectrum calculated for a given frequency range with error and a probability of the goodness-of-fit $Q$. The break frequency $\nu_{\mathrm{b}}$ is indicated

\begin{tabular}{llllllllll}
\hline PSR B & $\begin{array}{l}\text { Freq. range } \\
{[\mathrm{GHz}]}\end{array}$ & $\alpha_{1}$ & $\sigma_{\alpha 1}$ & $Q_{1}$ & $\alpha_{2}$ & $\sigma_{\alpha 2}$ & $Q_{2}$ & $\begin{array}{c}\nu_{\mathrm{b}} \\
{[\mathrm{GHz}]}\end{array}$ \\
\hline & & & & & & & & & \\
$0136+57$ & $0.4-4.9$ & -1.1 & 0.13 & $8.7 \mathrm{E}-02$ & -2.3 & 0.35 & $1.3 \mathrm{E}-02$ & 1.0 \\
$0226+70$ & $0.4-1.4$ & -0.5 & 0.24 & $5.8 \mathrm{E}-01$ & -4.0 & 0.85 & & 0.9 \\
$0301+19$ & $0.4-4.9$ & -0.9 & 0.38 & $5.0 \mathrm{E}-01$ & -2.3 & 0.34 & & & 0.9 \\
$0355+54$ & $0.4-23.0$ & -0.7 & 0.19 & $1.0 \mathrm{E}-02$ & -1.2 & 0.04 & $3.9 \mathrm{E}-01$ & 1.9 \\
$0540+23$ & $0.4-32.0$ & -0.3 & 0.14 & $8.3 \mathrm{E}-01$ & -1.6 & 0.09 & $6.0 \mathrm{E}-05$ & 1.4 \\
$0823+26$ & $0.4-14.8$ & -0.7 & 0.43 & $4.5 \mathrm{E}-01$ & -2.1 & 0.08 & $3.1 \mathrm{E}-05$ & 1.3 \\
$1237+25$ & $0.4-10.7$ & -0.9 & 0.19 & $7.4 \mathrm{E}-08$ & -2.2 & 0.25 & $2.5 \mathrm{E}-04$ & 1.4 \\
$1749-28$ & $0.4-10.7$ & -2.4 & 0.06 & $3.8 \mathrm{E}-02$ & -4.3 & 0.36 & $1.5 \mathrm{E}-01$ & 2.7 \\
$1800-21$ & $0.4-4.9$ & -0.2 & 0.07 & $1.3 \mathrm{E}-01$ & -1.0 & 0.32 & & & 1.4 \\
$1952+29$ & $0.4-10.7$ & -0.6 & 0.52 & $9.2 \mathrm{E}-01$ & -2.7 & 0.10 & $6.8 \mathrm{E}-01$ & 1.2 \\
$2011+38$ & $0.4-4.9$ & -0.9 & 0.13 & $1.5 \mathrm{E}-01$ & -1.9 & 0.10 & $7.3 \mathrm{E}-03$ & 1.4 \\
$2020+28$ & $0.4-32.0$ & -0.7 & 0.40 & $3.3 \mathrm{E}-01$ & -1.9 & 0.17 & $6.9 \mathrm{E}-01$ & 2.3 \\
$2021+51$ & $0.4-23.0$ & -0.8 & 0.20 & $1.8 \mathrm{E}-01$ & -1.5 & 0.07 & $2.0 \mathrm{E}-01$ & 2.6 \\
$2319+60$ & $0.4-10.6$ & -1.1 & 0.12 & $7.3 \mathrm{E}-07$ & -2.1 & 0.05 & $6.6 \mathrm{E}-03$ & 1.4 \\
$2324+60$ & $0.4-4.9$ & -1.2 & 0.12 & $5.8 \mathrm{E}-01$ & -2.5 & 0.27 & & & 1.4 \\
\hline
\end{tabular}

in three different observatories, the data seem consistent. In particular, the values obtained by Lorimer et al. (1995) and those from the Effelsberg radio-telescope at the same frequency are comparable (e.g., PSR B0740-28) ${ }^{1}$. We have calculated the spectral index for pulsars using the method described in Sect. 2.3. The results of this analysis are listed in Table 2. We found only 15 pulsars out of 167 whose spectral fit evidently required the two-powerlaw model (Table 3). In Fig. 1a we present distribution

\footnotetext{
1 All figures with spectra of 281 are available at: http://astro.ca.wsp.zgora.pl/olaf/paper1
}

of spectral indices $\alpha$ for pulsars with a simple power-law spectrum and in Figs. 1b and c for pulsars with a brokentype spectrum (see caption for explanation of $\alpha_{1}$ and $\alpha_{2}$ ). In Figs. $2 b$ and $c$ we also show two examples of two-powerlaw spectrum pulsars with the smallest and largest slope difference, respectively.

As detailed above, in order to reduce the effects of diffractive and refractive interstellar scintillations as well as possible intrinsic phenomena, we need a large number of measurements at a given frequency to obtain reliable pulsar spectra. We believe that our large sample of flux density measurements is capable of doing this over a wide frequency range, allowing an analysis of the spectral behaviour of pulsar radio emission. Our analysis shows that, in principle, pulsar spectra are described by a simple power law with the mean spectral index $\langle\alpha\rangle=-1.8 \pm 0.2$ (see Fig. 2a). We examined the data for any possible correlations between spectral index and rotation period $P$, spin-down rate $\dot{P}$, characteristic age $\tau$, polarization as well as profile type. In general no significant correlations were found but we have distinguished some interesting groups of objects which are discussed below:

(i) Very steep spectrum sources. This group of pulsars consists of objects with very steep spectra. Examples of such pulsars are the PSRs B0942-13, B0943+10 and $\mathrm{B} 1859+03^{1}$ with spectral indices of $-3.0,-3.7$ and -2.8 , respectively (see Table 2). Lorimer et al. (1995) suggested that older pulsars $\left(\tau \geq 10^{8} \mathrm{yr}\right)$ have steeper spectra, which is obviously not the case for B0943+10 and B1859+03 as these pulsars have characteristic ages of $4.910^{6} \mathrm{yr}$ 


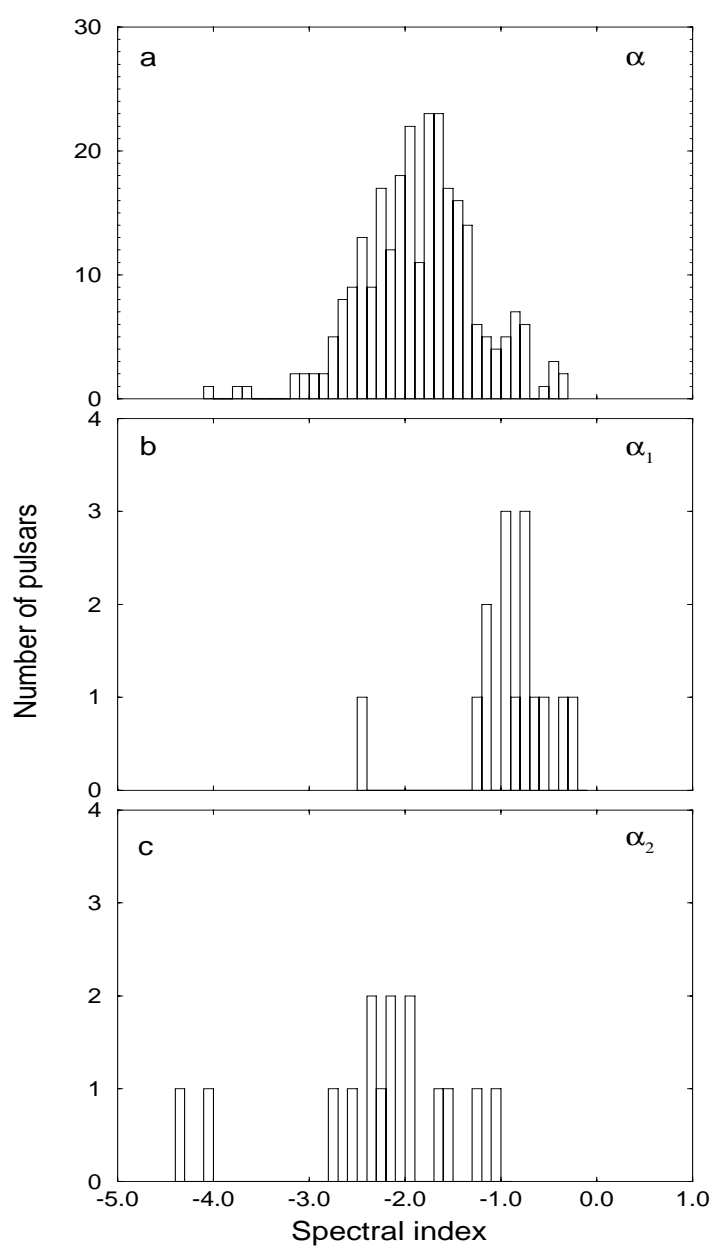

Fig. 1. a) The distribution of spectral index $\alpha$ for simple power law spectra, b) The distribution of spectral index $\alpha_{1}$ for the low frequency part of two-power-law spectra, c) The distribution of spectral index $\alpha_{2}$ for the high frequency part of two-power-law spectra

and $1.410^{6} \mathrm{yr}$, respectively. There is, of course, yet another exception, the Crab pulsar (PSR B0531+21), i.e. the youngest known radio pulsar with one of the steepest spectrum in our sample. These results provide evidence that there is no correlation between steepness of spectra and the characteristic pulsar age;

(ii) Flat spectrum sources. It was previously believed that pulsars have a steep spectra but the analysis of a large sample shows that there are pulsars with flat spectra over a wide frequency range. In this group there are pulsars which have almost flat spectra with $\alpha \geq-1.0$. Examples of such pulsars are B0144+59, B1750-24 and $\mathrm{B} 2022+50^{1}$ with spectral indices of $-1.0 \pm 0.04,-1.0 \pm 0.07$ and $-0.8 \pm 0.05$, respectively. Although Lorimer et al. (1995) suggested that younger pulsars have flat spectra, they also found that PSR B1952+29 possessed a flat spectrum and yet had a characteristic age of $3.410^{9} \mathrm{yr}$. This pulsar indeed has a flat spectrum in the frequency range from $400 \mathrm{MHz}$ to $1.4 \mathrm{GHz}$ but considering the whole
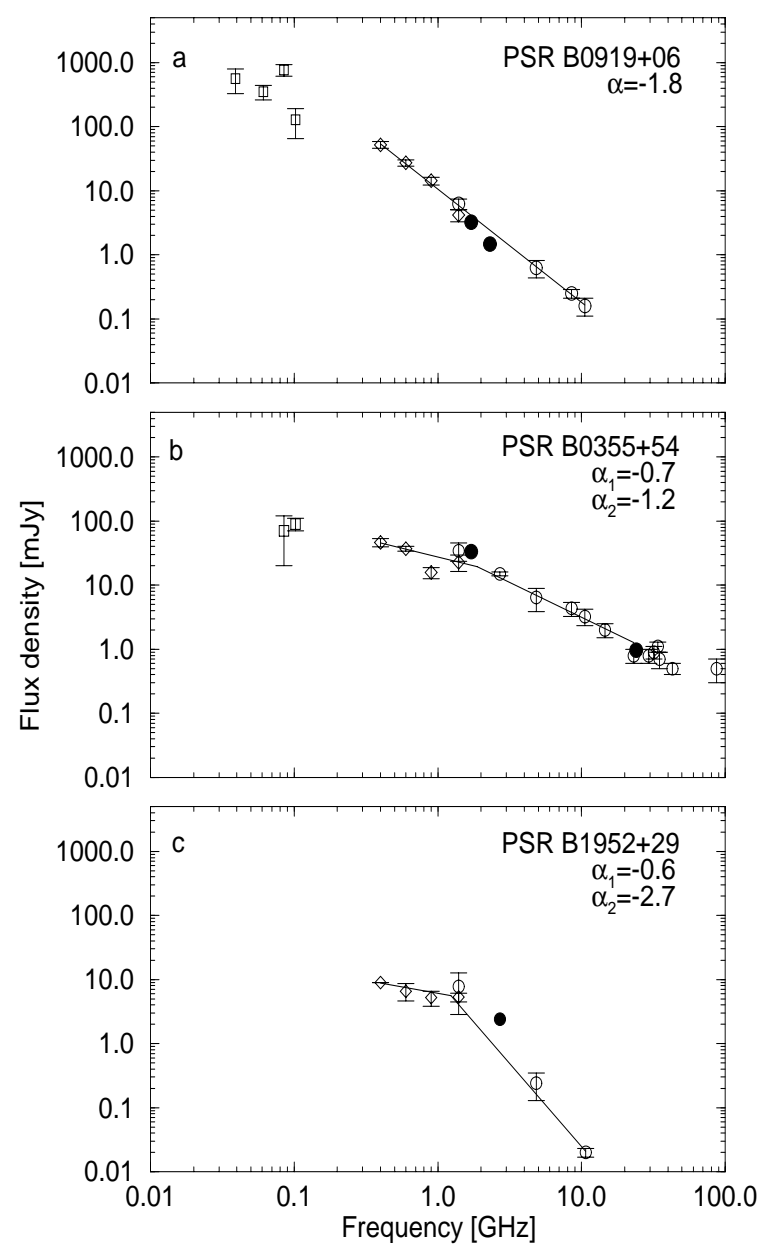

Fig. 2. a) Example of a typical spectrum with $\alpha=-1.8$, b) Example of the two-power law spectrum with the smallest difference in slopes $\left(\alpha_{1}=-0.7\right.$ and $\left.\alpha_{2}=-1.2\right)$, c) Example of the two-power law spectrum with the largest difference in slopes $\left(\alpha_{1}=-0.6\right.$ and $\left.\alpha_{2}=-2.7\right)$. Squares represent the measurements from Pushchino Radio Astronomical Observatory, diamonds represent measurements from Jodrell Bank and circles represent measurements from the Effelsberg Radiotelescope. Filled circles represent single measurements

frequency range from $400 \mathrm{MHz}$ to $10.7 \mathrm{GHz}$ its spectrum becomes a two-power-law one. A similar behaviour was observed for PSR B0540+23 ${ }^{1}$. It is possible that the pulsars with flat spectrum mentioned by Lorimer et al. (1995) may have a break in their spectrum at higher frequencies;

(iii) Sources with low-frequency turn-over. Spectra of many pulsars show a low-frequency turn-over at $\sim 100 \mathrm{MHz}$ (Sieber 1973; Izvekova et al. 1981). We have not fitted the turn-over points because of the gap in flux density measurements at frequencies between $100 \mathrm{MHz}$ and $300 \mathrm{MHz}$ and difficulties in determining the maximum frequency $\nu_{\max }$. We have found 2 pulsars in our sample which have a turn-over at unusually high frequency ( $\sim 1 \mathrm{GHz}):$ B1838-04 and B1823-13 (see Fig. 3). These are young pulsars and all belong to 
the 1800-21-class of pulsars, which was introduced by von Hoensbroech et al. (1998) to describe their unusual polarization properties. The PSR B1800-2 $1^{1}$ has a two power law spectrum, which may be also interpreted as a "broad form of turn-over";

(iv) Sources with high-frequency turn-up or flattening. There is a group of pulsars which have a possible turn-up or flattening in spectra at very high frequencies. Therefore we have not fitted the points above $23 \mathrm{GHz}$. In this group there are pulsars such as: B0329+54, B0355+54, B1929+10 and B2021+51 ${ }^{1}$ which were studied in detail by Kramer et al. (1996). There are also pulsars which may show a spectrum flattening already at $\sim 5 \mathrm{GHz}$. For example, the spectra of PSR B0144+59 and B2255+58 ${ }^{1}$. Recently, the idea of a spectral change at very high frequencies received a strong support from observations of the Crab pulsar. Moffet \& Hankins (1999) observed a clear flattening of its spectrum at realtively low frequency around $10 \mathrm{GHz}$, as compared with about $20 \mathrm{GHz}$ (Kramer et al. 1996);

(v) Sources with two power law spectra. We also recognized broken-type spectra (Sieber 1973; Malofeev et al. 1994), although there are only 15 definite two-power-law cases in our sample, showing so called break frequency between 0.9 and $2.7 \mathrm{GHz}$ (see Table 3) which divides the whole spectrum into two parts with considerably different slopes. This is a significantly smaller fraction (only about $10 \%$ ) than the reported $35 \%$ by Malofeev (1996). The distribution of spectral indices for broken-type pulsars is shown in Figs. 1b and c. The reduced fraction of twopower-law spectra pulsars can be only partly explained by selection effects possibly present in the Malofeev sample. In fact, many pulsars which were previously thought to demonstrate two-power-law spectra (Malofeev et al. 1994; Kramer et al. 1996; Xilouris et al. 1996) can be modelled by a simple power-law spectra in our sample. We believe that this can be largely explained by severe flux density variations (see Sect. 2.2) and the fact that the number of measurements included into a fit so far may have been too small (e.g. PSR B0628+281).

We note that Gil et al. (1994) and Malofeev (1996) suggested that PSR B1822-09 has a complex flux density spectrum. Our data do not confirm such a behaviour although some deviations from a simple power law seem to be present. Moreover, there are indeed some other pulsars which exhibit a rather unusual spectral behaviour (e.g. B0823+26, B0621-04, B0656+14 ${ }^{1}$ ). These objects may actually have a complex spectrum but we certainly need more and better data before we should consider them as a separate class of sources. Generally, pulsar spectra can be classified in two groups: out of 167 pulsars which have been studied over a wide frequency range (from $400 \mathrm{MHz}$ up to at least $5 \mathrm{GHz}$ ), the spectra of most pulsars can be modelled by a simple power law. About $10 \%$ of all pulsars require two power laws to fit the data. Table 4 and references therein clearly indicate that the spectra of

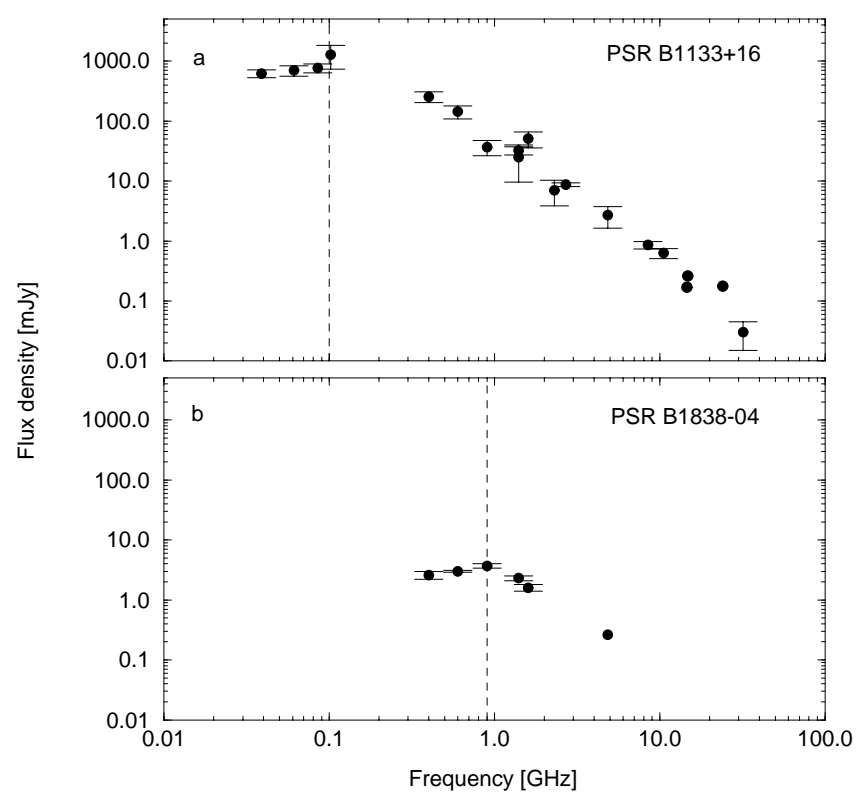

Fig. 3. a) Typical low frequency turn-over, b) an example of the unusual turn-over at around $1 \mathrm{GHz}$

slowly and fast rotating pulsars (i.e. millisecond pulsars) are indeed identical on the average. We note again, that the analysis of the main physical parameters of pulsars with unusual or two-power-law spectra has not shown any correlation, consistent with the results of Xilouris et al. (1996) and Malofeev (1996).

\section{Summary and conclusions}

The main conclusion of this paper is that the single power law spectrum is a rule and the two power law spectrum is a rare exception to this rule. One could therefore think that the nature of this exception is that the spectrum just becomes steeper starting from some, relatively high frequency. However, inspection of Fig. 1 indicates that this might not be a case. The distribution of $\alpha_{1}$ seems different from that of $\alpha$, meaning that in pulsars with two power law spectra the average spectral index $\left\langle\alpha_{1}\right\rangle$ is typically much larger than the average $\langle\alpha\rangle$ (Fig. 1b) and of course, the high frequency index $\left\langle\alpha_{2}>\right.$ is much smaller than $\langle\alpha\rangle$ (Fig. 1c). Thus, it seems that the two power law spectra are qualitatively different from the typical single power law spectra. In this paper we obtained spectral index for a large sample of pulsars in a wide frequency range (form $400 \mathrm{MHz}$ to $23 \mathrm{GHz}$ ). The average spectral index of pulsars $\langle\alpha\rangle$ with simple power-law spectrum in our sample is $-1.8 \pm 0.2$ which agrees with results obtained by other authors (see Table 4 ). The distribution of spectral indices is symmetric and almost Gaussian. The average indices for the broken-type spectra are $\left\langle\alpha_{1}\right\rangle=-0.9 \pm 0.5$ and $\left\langle\alpha_{2}\right\rangle=-2.2 \pm 0.9$, respectively, with a break frequency of $\left\langle\nu_{\mathrm{b}}>\approx\right.$ 1.5 GHz on the average. We have not found any 
Table 4. Spectral indices obtained by different authors

\begin{tabular}{crcl}
\hline $\begin{array}{c}\text { Spectral } \\
\text { index }\end{array}$ & $\begin{array}{r}\text { No. of } \\
\text { PSRs }\end{array}$ & $\begin{array}{c}\text { Freq. range } \\
{[\mathrm{GHz}]}\end{array}$ & References \\
\hline-1.6 & 27 & $0.1-10$ & Sieber 1973 \\
-1.9 & 20 & $0.1-30$ & Malofeev et al. 1994 \\
-1.6 & 280 & $0.3-1.6$ & Lorimer et al. 1995 \\
-1.7 & 284 & $0.1-10$ & Malofeev et al. 1996 \\
-1.8 & 32 & $0.3-4.9$ & $\begin{array}{l}\text { Kramer et al. 1998, 1999 } \\
\text { (millisecond PSRs) } \\
-1.7\end{array}$ \\
& 216 & $0.4-1.5$ & $\begin{array}{l}\text { Toscano et al. 1998 } \\
\text { (southern PSRs) }\end{array}$ \\
-1.9 & 19 & $0.4-1.5$ & $\begin{array}{l}\text { Toscano et al. 1998 } \\
\text { (millisecond PSRs) }\end{array}$ \\
-1.9 & 144 & $1.4-4.9$ & $\begin{array}{l}\text { Kijak et al. 1998 } \\
\text { this paper }\end{array}$ \\
\hline
\end{tabular}

correlations between spectral index and rotation period $P$, spin-down rate $\dot{P}$, characteristic age $\tau$, polarization and profile type for pulsars with both simple power law spectra and two-power-law spectra. We have found 2 young, nearly fully polarized pulsars which indicate turnover at unusually high frequency $(\sim 1 \mathrm{GHz})$. We have also found 15 pulsars which definitely require two-power-law spectra. The comparison of pulsar spectra analysis for slow and millisecond pulsars indicates that both groups have the same emission mechanism (Table 4).

Postscript files of spectra are available at http://astro.ca.wsp.zgora.pl/olaf/paper1 and our spectra are also presented in EPN Database at http://www.mpifr-bonn.mpg.de

Acknowledgements. We thank Christoph Lange for his help with our observations in August 1998. OM thanks the director of the MPIfR Prof. Dr. R. Wielebinski for invitation and support. OM and JK gratefully acknowledge several discussions with J. Gil in the course of this work. The authors also thank V.M. Malofeev for unpublished data at $102 \mathrm{MHz}$ and his helpful comments. This work was supported in part by the Polish State Committee for Scientific Research Grant 2 P03D 00819.

\section{References}

Bartel N., Sieber W., Wielebinski R., 1978, A\&A 68, 361 Foster R.S., Fairhead L., Backer D.C., 1991, ApJ 378, 687 Gil J.A., Jessner A., Kijak J., et al., 1994, A\&A 282, 45 Hoensbroech A. von, Xilouris K.M., 1997, A\&AS 126, 121

Hoensbroech A. von, Kijak J., Krawczyk A., 1998, A\&A 334, 571
Izvekova V.A., Kuzmin A.D., Malofeev V.M., Shitov Yu.P., 1981, ASS 78, 45

Kijak J., Kramer M., Wielebinski R., Jessner A., 1998, A\&AS 127,153

Kramer M., 1995, Ph.D. Thesis, High Frequency Observations of Pulsars, University of Bonn

Kramer M., Xilouris K.M., Jessner A., et al., 1996, A\&A 306, 867

Kramer M., Jessner A., Doroshenko O., Wielebinski R., 1997, ApJ 488, 364

Kramer M., Xilouris K.M., Lorimer D.R., et al., 1998, ApJ 501, 270

Kramer M., Lange C., Lorimer D.R., et al., 1999, ApJ 526, 957

Kuzmin A.D., Losovsky B.Ya., 2000, ASP Conf. Ser. 202, Kramer M., Wex N., Wielebinski R. (eds.)

Lorimer D.R., Yates J.A., Lyne A.G., Gould D.M., 1995, MNRAS 273, 411

Lorimer D.R., Jessner A., Seiradakis J.H., et al., 1998, A\&AS 128,541

Malofeev V.M., 1996, ASP Conf. Ser. 105, Johnson S., Walker M.A., Bailes M. (eds.)

Malofeev V.M., Gil J.A., Jessner A., et al., 1994, A\&A 285, 201

Malofeev V.M., Shishov V.I., Sieber W., et al., 1996, A\&A 308, 180

Malofeev V.M., Malov O., Shchegoleva N., 2000, Astron. Reports 44, 436

McLean A.I.O., 1973, MNRAS 165, 133

Moffet D.A., Hankins T.H., 1999, astro-ph/9904421

Morris D., Kramer M., Thum C., et al., 1997, A\&A 322L, 17

Nelder J.A., Mead R., 1965, Comp. J. 7, 308

Ommen van T.D., D'Alessandro F.D., Hamilton P.A., McCulloch P.M., 1997, MNRAS 287, 307

Press W.H., Teukolsky S.A., Vetterling W.T., Flannery B.P., 1996, Numerical Recipes in C. Cambridge University Press

Robinson B.J., Cooper B.F.C., Gardner F.F., Wielebinski R., Landecker T.L., 1968, Nat 218, 1143

Seiradakis J.H., Gil J.A., Graham D.A., et al., 1995, A\&AS 111, 205

Sieber W., 1973, A\&A 28, 237

Sieber W., Reinecke R., Wielebinski R., 1975, A\&A 38, 169

Sieber W., Wielebinski R., 1987, A\&A 177, 342

Slee O.B., Alurkar S.K., Bobra A.D., 1986, Aust. J. Phys. 39, 103

Stinebring D.R., Condon J.J., 1990, ApJ 352, 207

Thorsett S.E., 1991, ApJ 377, 263

Toscano M., Bailes M., Manchester R.N., Sandhu J.S., 1998, ApJ 506, 863

Wielebinski R., Jessner A., Kramer M., Gil J.A., 1993, A\&A 272,113

Xilouris K.M., Kramer M, Jessner A., Wielebinski R., Timofeev M., 1996, A\&A 309, 481 